\title{
A New Second-order Bistable Adaptive Stochastic Resonance Noise Reduction Method
}

\author{
Tang Rui ${ }^{1, a}$ \\ ${ }^{1}$ School of Mechanical Engineering, Panzhihua University, Panzhihua,China,617000 \\ a904200315@qq.com
}

Keywords: High performance composite components; Resonance noise reduction; Bistable adaptation

\begin{abstract}
Based on high performance composite components manufacturing process in the system of state monitoring and fault diagnosis is the key problem, established the mathematical model of a new type of second order bistable and proposes a adaptive stochastic resonance noise reduction method based on the model, has carried on the related experimental verification, the results show that the method has high computing precision and good detection result.
\end{abstract}

\section{Introduction}

There are important moving parts in intelligent manufacturing equipment of the high performance composite material components, such as motor, gear case and spindle, et c [1]. If they break down, equipments could stop movement, and which could even cause serious potential safety hazard and economic accident. Therefore, it is necessary to proceed to state detection and fault diagnosis for the key components of the manufacturing equipment of high performance composite material components [2]. When it works in a hostile environment or occured early breakdown, the vibration signal of breakdown will be coverd by the noise signal in the environment, so that the accuracy of fault diagnosis will be seriously affected [3]. Therefore, the vibration signal of the high performance composite component manufacturing equipment need be reduced noise at first. This paper will establish a kind of new high - order bistable stochastic resonance model, which is used to extract the weak signal characteristic [4].

\section{Bistable Stochastic Resonance Theory}

Stochastic resonance is a kind of phenomenon, in nonlinear system, weak signal will enhence and noise signal will significantly weaken when the small parameter signal and noise are interacting with each other. Many literatures have certified that the effectiveness of the nonlinear stochastic resonance method in weak signal analysis [5]. The following is a description of the stochastic resonance using a damping motion of a Brownian particle in a bistable potential well.

$$
\begin{aligned}
\frac{d x}{d t}=-U^{\prime}(x)+s(t) & +n(t) \\
U(x) & =-\frac{a}{2} x^{2}+\frac{b}{4} x^{4}
\end{aligned}
$$

where, $\mathrm{x}(\mathrm{t})$ is the system output, $\mathrm{s}(\mathrm{t})$ is periodic or aperiodic signal, $\mathrm{n}(\mathrm{t})$ is Gaussian white noise, and $\mathrm{U}(\mathrm{t})$ indicates the quartic symmetric potential function, its expression is as follows:(2.2)

The response of bistable stochastic resonance system can be realized by changing the noise density or system parameters. The basic bistable stochastic resonance system can use the quality of noise power spectral density $\operatorname{Sn}(\mathrm{f})$ lorentz distribution to explain further. The character of lorentz 
distribution is that most of the noise energy is concentrated in the low frequency region. This kind of energy concentration propels the formation of low-frequency driving components.

\section{New Second Order Bistable Model}

Most of the current approaches about bistable stochastic resonance are based on the model described by formula 2.1. From signal disposal perspective, the model is similar to the first-order low-pass filter. Compared with traditional low-pass smoothing, the stochastic resonance can not only filter out high frequency signal, but also increase the signal of the low frequency part because of the function of nonlinear term in the bistable model. Filter performance of filter can be refined by increasing the filter order. Similarly, in order to further improve the effect of stochastic resonance, it can be achieved by increasing the order of the bistable model[6]. Therefore, this paper established the second order bistable model, and its control equation is as follows:

$$
\frac{d^{2} x}{d t^{2}}+\frac{d x}{d t}=a x-b x^{3}+s(t)+n(t)
$$

Where, $a$ and $b$ are system parameters, $x(t)$ is output of system, $s(t)$ is input to system, and $n(t)$ is gaussian white noise. Through using this polymerous quadratic derivative, the system can further eliminate the noise. The nonlinear system represented by equation 3.1 can be solved by using the fourth-order runge-kutta method.

At first, condidering following noisy signal:

$$
y(t)=\sin (2 \pi \times 0.02 t)+n(t)
$$

Where, $\mathrm{N}(\mathrm{t})$ is a gaussian white noise with a standard deviation of 1.5. Sampled signal is $5 \mathrm{~Hz}$, signal length is 1000.In order to compare traditional bistable system with the bistable system proposed by this paper, this study uses same model parameters a and $b$, and the values are 1 and 2 respectively. The noisy signal is shown in figure1, the signal denoised by using the traditional bistable model is shown in figure 2 , and figure 3 shows the signal obtained by using the new bistable model. It can be shown from the comparison of figure 2 and figure 3, the signal noise is smaller and the curve is smoother in figure 3 .In addition, the signal-to-noise ratio of the signal obtained by the traditional model is $4.3 \mathrm{~dB}$, and the signal-to-noise ratio of the signal obtained by the new bistable model is $5.0 \mathrm{~dB}$. The signal-to-noise ratio is $5.0 \mathrm{~dB}$. The simulation results verified that the new system hasbetter noise reduction effect because of the existence of second order differential.

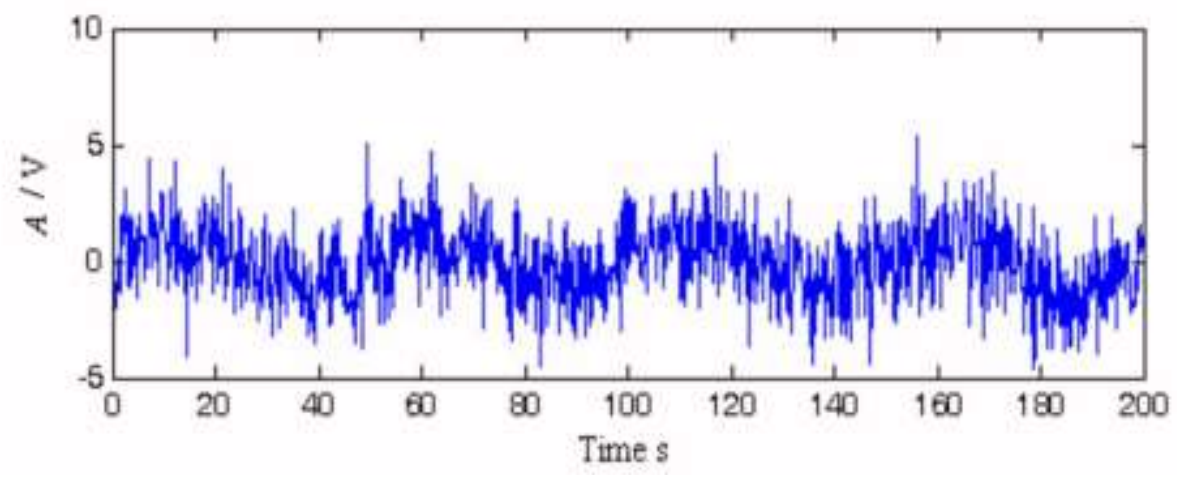

Figure 1. Sinusoidal waveform diagram of noisy signal. 


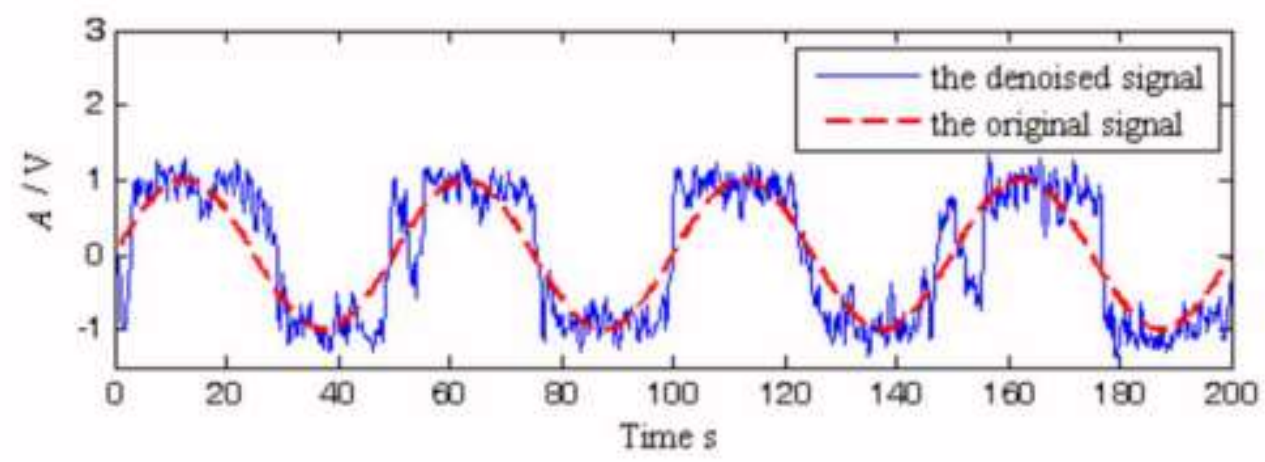

Figure2. Noise reduction signal obtained by traditional bistable model.

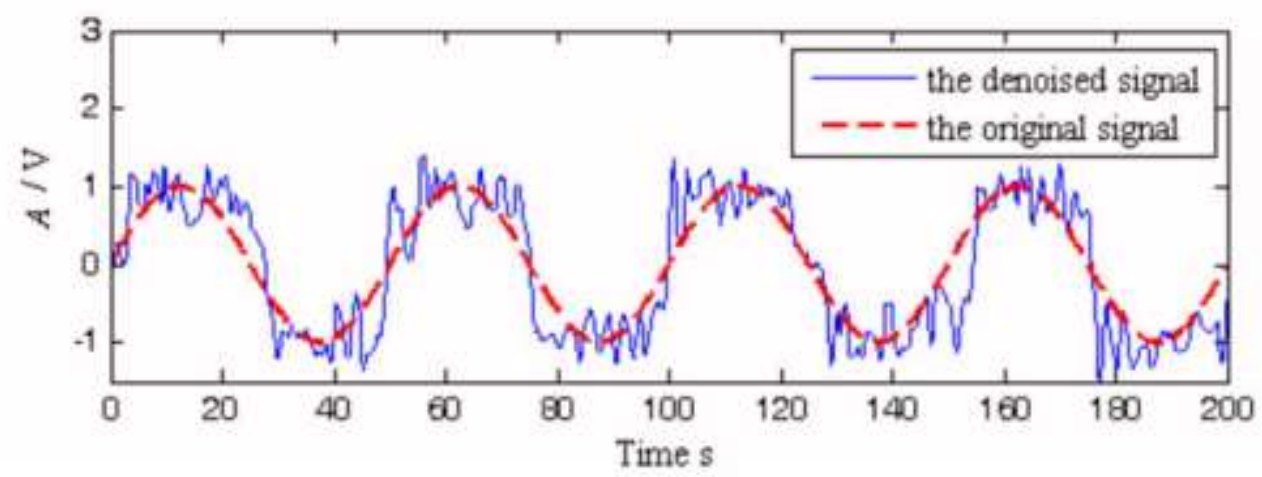

Figure.3. Noise reduction signal obtained by newl bistable model.

\section{Experimental Verification}

In order to verify the availability of the method which this paper proposed for the fault diagnosis of intelligent manufacturing equipment of high performance composite materials components, we analyzed the main shaft vibration signal of the driving device in manufacturing equipment of some high performance composite material component. The spindle has unbalanced fault, and its rotation frequency is $89.6853 \mathrm{~Hz}$ when picking up the signal. In order to generate random resonance phenomenon, a large sample frequency is adopted, and the sample frequency used is $40 \mathrm{kHz}$, the length of sampling data is 10240 . The time-domain plot and frequency-domain plot of the vibration signal collected are shown in figure 4 (a) and fig.4 (b) respectively. It can be seen from fig.4 (b), there is a clear line $a t f_{\mathrm{r}}$, but there is no visible line at the frequency doubling of rotation frequency because of the existence of strong noise. Therefore, it needs to use the adaptive stochastic resonance noise reduction method proposed in this chapter to process the signal. The frequency scale $\mathrm{R}$ is 4000 , and the decomposition layer is 6 . Through five iteration, the optimized test results are obtained. The time-domain waveform and frequency spectrum of signal after noise reduction are shown in fig. 5(a) and fig. 5 (b). It can be seen from fig.5 (b) that two distinct lines appeared at $2 f_{\mathrm{r}}$ and $3 f_{\mathrm{r}}$, but their amplitudes are smaller than the spectrum line at $f_{\mathrm{r}}$. Therefore, it can be judged that the spindle of drive device in high performance composite material components manufacturing equipment appeared unbalance fault. It is found that the spindle has eccentric quality after overhaul, it needs to proceed dynamic disposal to solve unbalance fault.. 
a

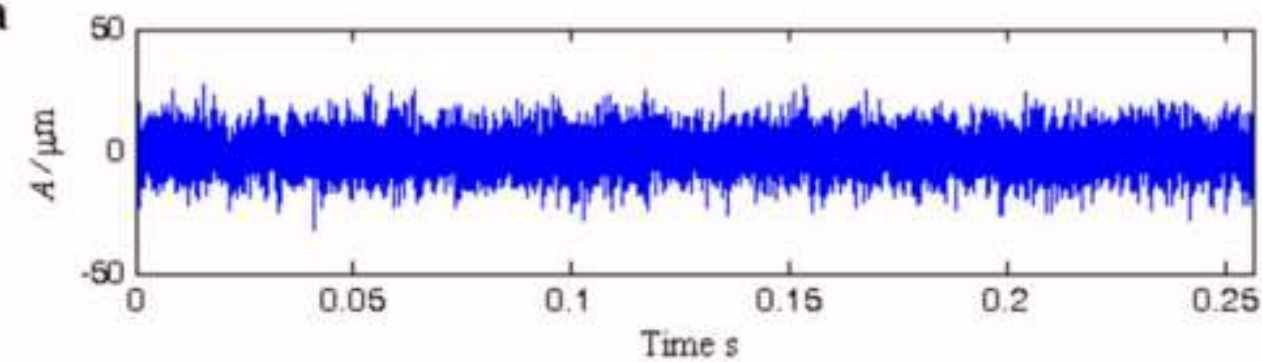

b

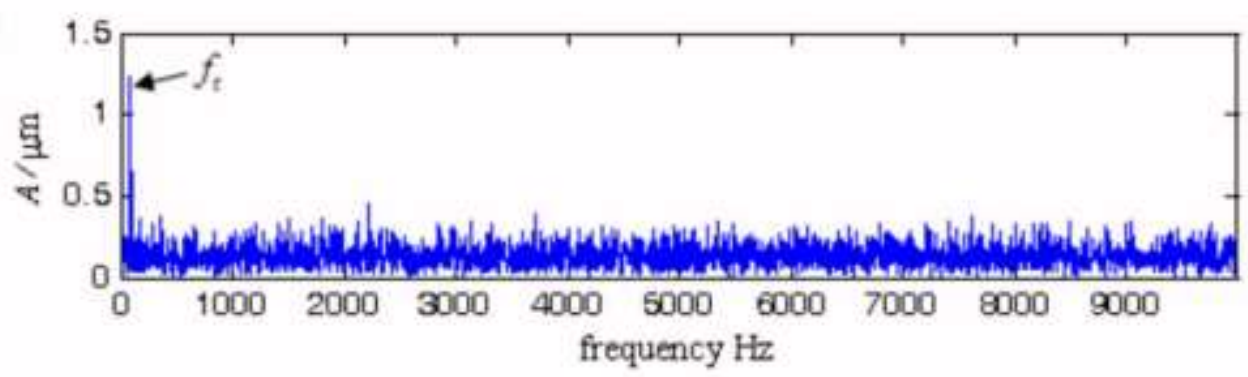

Figure.4. Spindle unbalanced fault vibration signal and frequency spctrum:(a)time-domain waveform;(b)frequency spctrum.

a

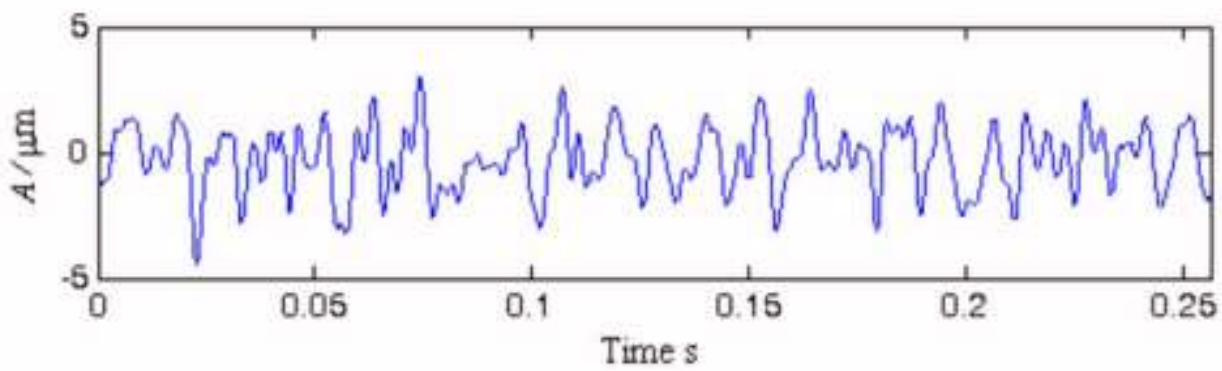

b

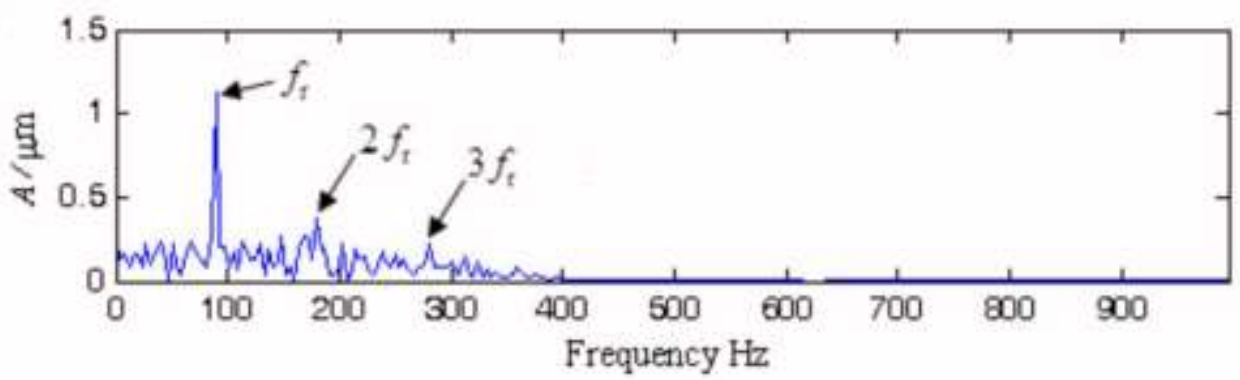

Figure.5. Spindle unbalanced fault vibration signal and frequency spctrum propsed by this paper:(a)time-domain waveform;(b)frequency spctrum

\section{Conclusion}

Aimed at the noise reduction problem of status monitoring signal of high performance composite materials component manufacturing equipment at the background of strong noise.this paper proposed a kind of new bistable adaptive stochastic resonance noise reduction method, which verified that the new stochastic resonance noise reduction method has very good detection effect and higher operation effect in weak signal extraction by the driver spindle unbalance fault diagnosis experiment of high performance composite material components.

\section{Reference:}

[1] R. Benzi, A. Sutera, A. Vulpiana. The mechanism of stochastic resonance [J]. Journal of 
Physics A: Mathematical and General, 1981, 14(11): 453-457.

[2] L. Gammaitoni, P. Hanggi, P. Jung, F. Marchesoni. Stochastic Resonance [J]. Reviews of Modern Physics, 1998, 70(1): 223-287.

[3] H. Gang, G. Nicolis, C. Nicolis. Periodically forced Fokker-Planck equation and stochastic resonance [J], Physical ReviewA, 1990, 42 (4): 2030-2041.

[4] Qin Yi, Wang Teng, He Qiyuan, Ren Bing Application of High Density Wavelet Transform in Rolling Bearing Compound Fault Diagnosis. Journal of Chongqing University, 2013, 36 (3): 13-19.

[5] LENG Yong-gang, WANG Tai-yong. Numerical Study on the Extraction of Weak Signal from Random Noise by Quadratic Sampling for Stochastic Resonance [J] .Acta Physica Sinica, 2003, $52,10,2432-2437$.

[6] V. Kohar, K .Murali, S. Sinh. Enhanced logical stochastic resonance under periodic forcing [J], Communications in Nonlinear Science and Numerical Simulation, 2014, 19 (8): 2866-2873.

[7] Peng L. Individual Vision and Peak Distribution in Collective Actions [J]. Communications in Nonlinear Science and Numerical Simulation. 2017, 47: 238-252. 